12. Tanner JM, Goldstein H, Whitehouse RH. Standards for children's height at ages 2-9 years allowing for height of parents. Arch Dis Child 1970; 45: 755-62.

13. Raben MS. Treatment of a pituitary dwarf with human growth hormone. J Clin Endocrinol Metab 1958; 18: 901-3.

14. Ranke Michael B, Lindberg Anders. Observed and Predicted Growth Responses in Prepubertal Children with Growth Disorders: Guidance of Growth Hormone Treatment by Empirical Variables. J Clin Endocrinol Metab 2010; 95:1229-37.

15. Cowell CT. Short stature. In: Clinical Paediatric Endocrinology $3^{\text {rd }}$ edition $1995,136-172$. Edited by Charles G.D. Brook. Blackwell Science Ltd. University Press, Cambridge.
16. de Mel T, Warnasooriya N, Fonseka C. Growth hormone deficiency in Sri Lanka: a preliminary study. Ceylon Med $J$ 1991; 36: 95-7.

17. Gamage KKK, Mangaleswaran S, Galappaththy SAL, et al. Growth hormone therapy for short stature in adolescents - the experience in the University Medical Unit, National Hospital of Sri Lanka. Sri Lanka J Diabetes, Endocrinol Metab 2014; 4: 49-55.

18. de Silva KSH, de Zoysa P. Assessment of the psychological status of children with growth hormone deficiency and their parents. Ceylon Med J 2011; 56: 59-61.

19. Bajpai Anurag, Kabra Madhulika, Gupta Arun Kumar, Menon PSN. Growth Pattern and Skeletal Maturation Following Growth Hormone Therapy in Growth Hormone Deficiency: Factors Influencing Outcome. Indian Pediatr 2006; 43: 593-99.

\title{
A comparison of endometrial thickness following augmentation of ovulation with clomifene citrate or letrozole in women with ovulatory infertility
}

\author{
T S Palihawadana ${ }^{1}$, P S Wijesinghe ${ }^{1}$, H R Seneviratne ${ }^{2}$
}

(Index words: letrozole, augmentation of ovulation, endometrial thickness)

\begin{abstract}
Objectives To compare endometrial thickness following augmentation of ovulation with clomifene (CC) and letrozole and to assess the rate of multi-follicle development and the FSH fluctuations during the stimulated cycles.

Methods A comparative study was carried out at the infertility clinic of Faculty of Medicine, University of Kelaniya, Sri Lanka. Each arm included 25 participants. They received either $100 \mathrm{mg}$ of CC or $2.5 \mathrm{mg}$ of letrozole per day for 5 days starting from 2 nd day of the cycle. The fluctuation of FSH in the follicular phase of the cycle, the endometrial thickness and the number of pre-ovulatory follicles on the day of detecting a mature follicle, were measured.
\end{abstract}

Results Treatment with letrozole resulted in a smaller rise in the follicular phase FSH, which was shorter in duration than with CC. Also it resulted in a higher mean endometrial thickness at the time of detecting a mature follicle [9.89 $(\mathrm{SD}=2.02) \mathrm{mm}$ vs. $8.58(\mathrm{SD}=1.32) \mathrm{mm} ; p=0.021]$. Augmentation of ovulation with $\mathrm{CC}$ showed a trend towards multi-follicle development compared to letrozole.

Conclusions This study suggests that letrozole is superior to $\mathrm{CC}$ in augmentation of ovulation in terms of endometrial growth and mono-follicle development. However more studies are needed to assess how these differences affect the treatment outcome before it is recommended for clinical use.

Ceylon Medical Journal 2015; 60: 48-52

\section{Introduction}

Infertility is known to affect around one in six couples worldwide [1]. In some no abnormality can be found, and are therefore termed as having unexplained infertility. The prevalence of male factor infertility is thought to be around $20-40 \%$ while unexplained infertility is estimated to be around $10-28 \%$ [2-5]. Augmentation of ovulation is widely used to improve fertility in women with unexplained infertility and also as an adjunct to intrauterine insemi-nation in male factor infertility. Clomifene citrate (CC) is the commonest anti-oestrogen used for induction and augmentation of ovulation. A third generation aromatase inhibitor letrozole is a newer therapeutic agent proposed for same purposes. It is proposed that letrozole has some advantages over CC

${ }^{1}$ Department of Obstetrics and Gynaecology, Faculty of Medicine, University of Kelaniya and ${ }^{2}$ Department of Obstetrics and Gynaecology, Faculty of Medicine, University of Colombo.

Correspondence: TSP, e-mail: <thilipali@kIn.ac.lk>. Received 22 May 2014 and revised version accepted 13 October 2014. 
since it does not cause down regulation of oestrogen receptors and confer no anti-oestrogenic effects on the endometrium [6].

Mono-follicle development is a physiological phenomenon tightly controlled by hormone feedback mechanisms brought about by limiting the FSH rise in the early follicular phase beyond a critical level (FSH threshold) to a very limited time interval (FSH window). Interference with this feedback mechanism could result in multiple follicle development, which would result in multiple pregnancies, with its adverse consequences to both the mother and the offspring. Since letrozole therapy does not interfere with these feedback mechanisms of the hypothalamo-pituitary-ovarian axis, its effect resembles physiological FSH fluctuations more closely than CC therapy [6].

This study was aimed at comparing the two therapeutic agents, $\mathrm{CC}$ and letrozole, with regard to $\mathrm{FSH}$ fluctuations in the follicular phase, the effects on the endometrial thickness and multiple follicle development during augmentation of ovulation.

\section{Methods}

This study was carried out at the University Infertility Unit of the North Colombo Teaching Hospital, Ragama, Sri Lanka, in 2009 among couples seeking treatment for infertility. A comparative study was carried out with two treatment groups. The study participants were those with confirmed regular ovulation that required augmentation of ovulation according to the clinical management protocols used in the unit. Their FSH, LH, prolactin, TSH and serum testosterone levels were within normal limits.

Sample size calculation was calculated to demonstrate a difference in the endometrial thickness between the two groups. Previous studies have described endometrial thickness after ovulation induction with $\mathrm{CC}$ of $8.5 \mathrm{~mm}$ (SD 1.15) and with letrozole $7.5 \mathrm{~mm}$ (SD 0.2) [7,8]. To demonstrate a difference of $0.8 \mathrm{~mm}$ or more with a significant level of $5 \%$ and a study power of $80 \%$, a sample size of 18 in each group. There was no data available on possible drop-outs and those who fail to ovulate in the index cycle, and therefore could not be included in the analysis, we planned to include 25 participants in each study arm. The study participants were randomised to treatment groups using opaque envelopes to receive either letrozole or CC. The recruitment was done on the second day of a spontaneous menstrual cycle and a baseline transvaginal ultrasound scan was performed to assess ovarian morphology. Treatment with either letrozole or $\mathrm{CC}$ was commenced on the second day of the menstrual cycle according to the allocation.

Those in the CC treatment group received 100 $\mathrm{mg}$ per day for five days and women in the other group received $2.5 \mathrm{mg}$ of letrozole per day for five days. The FSH assays were carried out on the $2^{\text {nd }}, 5^{\text {th }}$ and the $9^{\text {th }}$ day of the menstrual cycle and serum oestradiol levels were measured on the $5^{\text {th }}$ day and on the day of detecting a mature follicle. Transvaginal ultrasound for follicle tracking was commenced on the $9^{\text {th }}$ day of the menstrual cycle and was continued till a mature follicle $(>18 \mathrm{~mm})$ was observed. The endometrial thickness was measured on the $9^{\text {th }}$ day of the menstrual cycle and on the day a mature follicle was observed. It was measured at the greatest diameter perpendicular to the mid-sagittal plane in the fundal region, including both layers of the endometrial cavity as described in previous studies [9]. The number of pre-ovulatory follicles ( $>15 \mathrm{~mm}$ ) was counted on the day of detecting a mature follicle. All ultrasound assessments were done by a single investigator (TP), to minimize any inter-observer variability. The rest of the clinical management was according to the individual management plan for each participant. The baseline characteristics and all other quantitative data were compared between the two groups using the student t-test and multifollicle development was compared using the odds ratio and the chi test. Approval for the study was obtained from the Ethics Review Committee of the Faculty of Medicine, University of Kelaniya. The trial was registered with the Clinical Trials Registry of the Sri Lanka Medical Association. Written informed consent was obtained from all patients.

\section{Results}

The two study groups were comparable in baseline characteristics. They did not show any significant difference in mean age, duration of infertility or mean BMI. The mean ovarian volume and the mean antral follicle count measured on an early follicular phase scan were similar in the two groups. The FSH level done on the second day of the menstrual cycle was also similar between groups. The comparison of above characteristics is shown in table 1 .

Ovulation was confirmed in 22 participants in the group who received CC. One participant in this group failed to develop mature follicles while in another two participants unruptured follicles were noted on follicle tracking. In the group that received letrozole, ovulation was noted in 23 participants while non-development of follicles and an unruptured mature follicle was noted in one subject each.

The mean fluctuations of FSH from the $2^{\text {nd }}$ to $9^{\text {th }}$ day of the treatment cycles in the two groups are shown in figure 1 . The FSH rise on the $5^{\text {th }}$ day of the menstrual cycle was higher among those who received CC [10.69 $(\mathrm{SD}=4.66) \mathrm{IU} / 1$ compared to those who received letrozole $8.73(\mathrm{SD}=2.09) \mathrm{IU} / \mathrm{l} ;(p=0.061)$. It remained higher also on the $9^{\text {th }}$ day of the menstrual cycle among subjects who received $\mathrm{CC}[7.28(\mathrm{SD}=4.24) \mathrm{IU} / 1$ compared with letrozole $6.45(\mathrm{SD}=2.52) \mathrm{iu} / 1(p=0.404)$. However, the difference was not statistically significant on both occasions.

The oestradiol level was significantly higher in those who received CC $342.03(\mathrm{SD}=304.44) \mathrm{pg} / \mathrm{ml}$ than those 
Table 1. Comparison of the basic characteristics between two treatment groups

\begin{tabular}{|c|c|c|c|}
\hline Characteristic & $\begin{array}{c}\text { Clomifene } \\
n=25\end{array}$ & $\begin{array}{c}\text { Letrozole } \\
n=25\end{array}$ & Significance \\
\hline Age of the subjects; mean(SD) years & $30.08(3.26)$ & $30.40(3.92)$ & $p=0.755$ \\
\hline Duration of infertility; mean(SD) months & $30.3(24.6)$ & $34.4(23.32)$ & $p=0.553$ \\
\hline $\mathrm{BMI} ;$ mean(SD) kg/m² & $23.2(3.23)$ & $22.8(2.73)$ & $p=0.716$ \\
\hline Mean ovarian volume; mean(SD) $\mathrm{cm}^{3}$ & $8.01(2.95)$ & $6.85(3.88)$ & $p=0.239$ \\
\hline Mean antral follicle count; mean(SD) & $9.06(1.32)$ & $6.84(4.07)$ & $p=0.798$ \\
\hline Day 2 FSH level; mean(SD) iu/l & $8.75(3.19)$ & $8.79(2.50)$ & $p=0.960$ \\
\hline
\end{tabular}

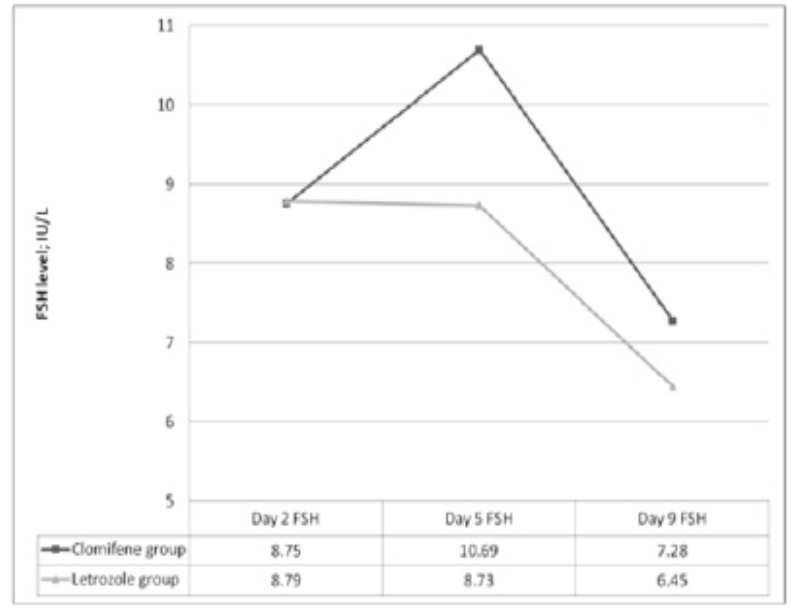

Figure 1. The fluctuation in the FSH level during the follicle phase of the cycle among participants who received clomifene citrate and letrozole.

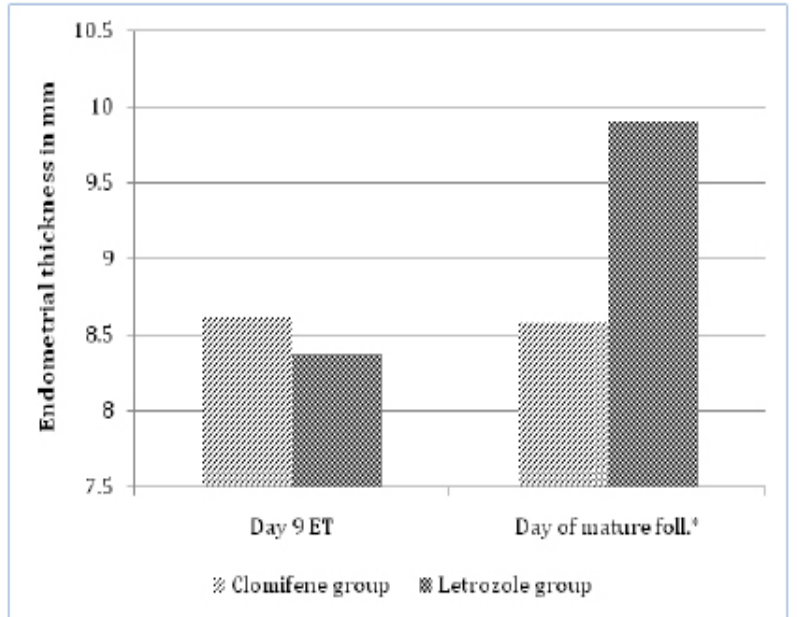

* Denotes a statistically significant difference

Figure 2. Comparison of endometrial thickness in the two treatment groups on $9^{\text {th }}$ day of the cycle and the day of detecting a mature follicle. who received letrozole $80.14(\mathrm{SD}=70.49) \mathrm{pg} / \mathrm{ml} ; p<0.001$ on the $5^{\text {th }}$ day of the menstrual cycle and it remained higher on the day of detecting a mature follicle [897.06 (661.21) $\mathrm{pg} / \mathrm{ml}$ vs. 143.55 (79.56) $\mathrm{pg} / \mathrm{ml} ; p<0.0001]$.

The mean endometrial thickness did not show a significant difference on the $9^{\text {th }}$ day of the menstrual cycle between those who received CC $8.61(\mathrm{SD}=1.21)$ $\mathrm{mm}$ and those who received letrozole 8.37 ( $\mathrm{SD}=$ $2.13) \mathrm{mm}$; $(p=0.666)$. However, on the day of detecting a mature follicle, it was significantly lower among those who received $\mathrm{CC} 8.58(\mathrm{SD}=1.32) \mathrm{mm}$ compared to those who received letrozole $9.89(\mathrm{SD}=2.02) \mathrm{mm},(p=0.021)$. This is shown in figure 2 .

Development of two or more pre-ovulatory follicles $(>15 \mathrm{~mm})$ was noted in $13(52 \%)$ participants who received $\mathrm{CC}$ and in $9(36 \%)$ participants who received letrozole (OR 1.93; 95\% CI 0.63 - 5.83).

\section{Discussion}

This study demonstrated a significant difference in the thickness of the endometrium on the day of detecting a mature follicle. Those who received letrozole had a thicker endometrium than those who were treated with CC. Though multifollicle development was observed more frequently with $\mathrm{CC}$, it was not statistically significant. Similarly, the higher FSH rise and longer period of elevation of FSH among those who received CC compared to those who received letrozole, was not statistically significant.

Although CC is the most commonly used therapeutic agent for augmentation of ovulation worldwide, its limitations have been well recognised. Those include the anti-oestrogenic effects on the developing endometrium and the multi follicle development leading to multiple pregnancies [10]. Letrozole, a third generation aromatase inhibitor, is a newer therapeutic agent that has been proposed for this indication. It acts by inhibiting the aromatisation, which in turn reduces the production of oestrogen from all sources. This will release the HPO axis from oestrogen negative feedback [6].

We could not find a statistically significant difference 
in FSH rise in the early follicle phase of the cycle or the duration of its elevation between the two drugs. The excessive rise in FSH seen with $\mathrm{CC}$ is attributed to the prolonged loss of negative feedback on FSH secretion brought about by the depletion of oestrogen receptors by the drug. The longer half-life of the drug ( 5 days) is thought to be responsible for the persistent elevation of FSH in the follicular phase. Letrozole, on the other hand has a shorter duration of action as it does not deplete the oestrogen receptors. Also the shorter half-life of 45 hours helps to minimise the duration of FSH rise [11]. These properties of letrozole would minimise the interruption of the 'FSH threshold' and the 'FSH window' that is seen in physiological states thus preventing multiple follicle development. Though the number of dominant follicles in the two treatment groups was not significantly different $52 \%$ of those who received CC developed two or more follicles compared to $36 \%$ among those were treated with letrozole.

The proliferation of the endometrium was significantly more among women who received letrozole compared to those that received CC. This difference is attributed to the lack of negative anti-oestrogenic effects in letrozole, which are known to occur with any therapeutic agent with anti-oestrogenic properties [12]. A similar finding has been described in a previous study where letrozole and $\mathrm{CC}$ were used as an adjuvant for FSH in induction of ovulation [13]. However, the endometrial thickness in both treatment groups in our study was within the clinically acceptable levels. It can be argued that thickness of the endometrium is only an indicator of the development of the endometrium and avoidance of anti-oestrogens during its development will make it more favourable for implantation [14]. However, whether this difference in the thickness and associated effects seen with letrozole confer an added advantage in achieving pregnancy needs to be further evaluated. Such studies should be designed with pregnancy taken as an outcome measure. Furthermore, the recently published NICE clinical guidance on management of infertility by the National Institute of Clinical Excellence, has cautioned on use of letrozole as a therapeutic agent till more safety data is available [15]. This recommendation was based on two facts. Firstly, the safety of lerozole in ovulation induction has not been well established due to our limited experience with the drug and the existing evidence is inconclusive. Secondly, the current evidence on efficacy does not show a clear advantage for letrozole over CC as a first line ovulation induction agent to justify its use in the absence of proven safety. This highlights the need for more clinical trials of the drug and it should not be used outside clinical trials at present.

We recognised two limitations of this study. Firstly, since we have taken ovulation as the end point outcome we were unable to study the effect of these therapeutic agents on the pregnancy rates. As the participants in this study were not controlled for factors such as seminal parameters and clinical management protocols, pregnancy could not be taken as an outcome measure. The other limitation is that a smaller dose of letrozole $(2.5 \mathrm{mg})$ was used in this study. New evidence has emerged that a higher dose of letrozole $(5 \mathrm{mg})$ is more effective in ovulation induction [16]. The effects of higher doses of letrozole needs to be further evaluated.

In conclusion, this study demonstrates that letrozole results in a significantly higher endometrial thickness and a lower incidence of multi-follicle development. Both these are desirable effects in augmentation of ovulation. Therefore, it is proposed that further well-controlled studies be carried out with pregnancy as the outcome measure to see its efficacy in clinical use.

\section{Acknowledgements}

The study was funded by a research grant from the National Science Foundation of Sri Lanka. (Grant No: RG/2007/HS/08).

\section{Conflicts of interest}

We declare that there are no conflicts of interest.

\section{References}

1. World Health Organization, Infertility: A tabulation of available data on prevalence of primary and secondary infertility. Geneva, WHO Programme on Maternal and Child Health and Family Planning, Division of Family Health 1991.

2. Balen AH, Rutherford AJ. Management of infertility. BMJ 2007; 335: 608-11.

3. Evers J. Female subfertility. Lancet 2002; 360: 151-9.

4. Adamson GD, Baker VL. Subfertility: causes, treatment and outcome. Best Practice \& Research: Best Pract Res Clin Obstet Gynaecol 2003; 17: 169-85.

5. Hull MGR, Glazeber CMA, Kelly NJ, et al. Population study of causes, treatment and outcome of infertility. BMJ 1985; 291: 1693-7.

6. Casper RF, Mitwally MFM. Aromatase inhibitors for ovulation induction. J Clin Endocrinol Metab 2006; 91: 760-71.

7. Harith S, Rajagopalan G. Follicular growth, endometrial thickness and serum oestradiol levels in spontaneous and clomiphene citrate-induced cycles. Int J Gynecol Obstet 2003; 81: 287-92.

8. Quintero RB, Urban R, Lathi RB, Westphal LM, Dahan $\mathrm{MH}$. A comparison of Letrozole to gonadotropins for ovulation induction, in subjects who failed to conceive with clomiphene citrate. Fertil Steril 2007; 88: 879-85.

9. Zaidi J, Campbell S, Pittrof R, Tan SL. Endometrial thickness, morphology, vascular penetration and velocimetry in predicting implantation in an in vitro fertilization program. Ultrasound Obstet Gynecol 1995; 6: 191-8.

10. Fauser BCJM, Heusden AMV. Manipulation of human ovarian function: Physiological concepts and clinical consequences. Endocr Rev 1997; 18: 71-106. 
11. Casper RF. Aromatase inhibitors in ovarian stimulation. $J$ Steroid Biochem Mol Biol 2007; 106: 71-5.

12. Lambalk CB, Huirne JAF, Macklon NS, Fauser BCJM, Homburg R. Ovulation induction with clomiphene or gonadotropins. International Congress Series 2005; 1279: 16-23.

13. Barroso G, Menocal G, Felix H, Roja-Ruiz JC, Arslan M, Oehninger S. Comparison of the efficacy of the aromatase inhibitor Letrozole and clomiphene citrate as adjuvants to recombinant follicle-stimulating hormone in controlled ovarian hyperstimulation: a prospective, randomized, blinded clinical trial. Fertil Steril 2006; 86: 1428-31.
14. Dickey RP, Holtkamp DE. Development, pharmacology and clinical experience with clomiphene citrate. Hum Reprod update 1996; 2: 483-506.

15. National Institute of Clinical Excellence. Ovulation disorders. Fertility: assessment and treatment for people with fertility problems. London, RCOG press 2013: 139182.

16. Biljan MM, Tan SL, Tulandi T. Prospective randomized trial comparing the effects of 2.5 and $5.0 \mathrm{mg}$ of Letrozole on follicular development, endometrial thickness and pregnancy rate in patients undergoing super-ovulation. 